\title{
Automatic localization of Common Carotid Artery in ultrasound images using Deep Learning
}

\author{
Dina A. Hassanin ${ }^{1}$, Mahmoud khaled Abd-Ellah ${ }^{* 2}$, Ashraf A. M. Khalaf ${ }^{3}$ and \\ Reda R. Gharieb ${ }^{4}$ \\ ${ }^{1}$ Electrical Engineering Department, Egyptian Academy for Engineering and Advanced Technology, Egypt \\ ${ }^{2}$ Electronics and Communications Department, Al-Madina Higher Institute for Engineering and \\ Technology, Giza, Egypt \\ ${ }^{3}$ Electrical Engineering Department, Faculty of Engineering, Minia University, Egypt \\ ${ }^{4}$ Electrical Engineering Department, Faculty of Engineering, Assiut University, Egypt \\ *corresponding author E-mail: mahmoudkhaled@ieee.org
}

\begin{abstract}
Accurate and automatic localization of the common carotid artery (CCA) is extremely important because the narrowing of the CCA is a silent disease. CCA disease doesn't cause any symptoms in its early stages, and people don't realize that they usually have a problem until they have a stroke. A stroke occurs when the brain doesn't receive enough blood for a long time. Brain damage from a stroke can lead to loss of speech or vision, and major strokes can cause death. In this paper, we proposed various techniques to localize the CCA in transverse section ultrasound (US) images using deep learning. First, we applied preprocessing to the images in the dataset before detecting the bounding box containing the CCA. We used a faster regional proposal convolutional neural network (Faster R-CNN) to detect the rectangular region (bounding box) around the CCA. Then we applied various localization techniques to localize the CCA in the US images. The proposed method has been performed on ultrasonic transverse images of the signal processing (SP) Lab. We compared our results with the clinicians' circles obtaining a great match between them. The accuracy of the bounding box detection was 97.5 and a Jaccard similarity of $90.86 \%$ between our proposed system and the clinicians' manual circles. Our proposed system has shown results that outperform other systems in Literature.
\end{abstract}

Keywords: Common Carotid Artery, Deep Learning, ultrasound images, Convolutional neural network, detection of CCA, Localization of CCA

\section{INTRODUCTION}

Carotid arteries are known as the main blood vessels in the neck that supply blood to the brain, neck, and face[1]. The carotid artery obstruction occurs due to high cholesterol and the accumulation of fatty substances on the walls of blood vessels, causing artery narrowing that impedes blood flow to the brain and artery hardens. The narrowing or blockage of the arteries in the neck area may impede or stop the supply of blood to the brain, which leads to the occurrence of strokes[2]. Symptoms of narrowing of the arteries in the neck do not appear at the beginning of the disease and the symptoms begin to appear after a stroke. Therefore, it is necessary to make regular visits to the vascular specialist to detect problems early, especially for diabetics, smokers, and heart patients, starting from the age of 50 years. Stroke is the second most important cause of death in the world, after heart disease. Every year, 15 million people worldwide have a stroke, 5 million of them die, and another 5 million remain permanently disabled[3].

Received:14 Novamber, 2020, Accepted:16 Novamber, 2020
Automatic and accurate localization of the carotid artery is a major and important step in the early detection of carotid artery disease and the application of the necessary medical intervention without entering into health complications and treatment before the occurrence of a stroke[4].

US imaging is used as an expensive and painless noninvasive test in evaluating blood flow through the carotid arteries, by bouncing high-frequency ultrasound from the artery to create images. Doctors use ultrasound to check for carotid arterial injuries, or to monitor certain treatments given in the arteries, or to detect blood clots or stroke. Doppler US helps assess stroke risk, to identify and evaluate narrowing or blockages in the arteries in the neck and head. This procedure is useful for evaluating people who have had a stroke and who have risk factors for atherosclerosis but no symptoms[5].

Some studies have been proposed to localize and segment the CCA in transverse mode images. Yang et al.[6] have been segmented the CCA using histogram equalization, edge detection, nonlinear filtering, and 
Vol. 40, No. 2. July 2021

morphological operations as post-processing. They used 180 US images of the CCA obtained from three patients captured by a Philips/ATL HDI US machine. They obtained a JSC of $70.5 \%$ by the rapprochement between their contours and clinician's manual results. A hybrid semi-automated segmentation model is proposed to contour the CCA using the GVF-Snake model and morphological operations by Yang et al.[7].Their dataset contains 110 CCA slices captured by a Philips US machine with a frequency of 8.5 MHZ using a linear transducer. The comparison between their proposed model and the physician's results gives a JSC of $90.2 \%$.

Radek Benes et al.[8], have suggested a technique to localize the CCA depend on the grammar-guided genetic programming. Their collected dataset consists of 68 video sequences where the CCA centroid was marked for 25:45 aged volunteers. The authors obtained a resulting success of $82.7 \%$ for their proposed system by applying the validation process with expert results. Shivendra Singh et al.[9] have applied 3 machine learning models to localize the CCA as Logistic Regression, Support Vector Machine, and Adaboost classifier based on features selection. The best results were performed by the Adaboost classifier which reaches a localization accuracy of $91.66 \%$. Their dataset contains 100 ARTSENS images dividing as 60 images for training and 40 images for testing.

A segmentation algorithm of the CCA based on the modified Dynamic Programming and canny detector proposed by Hamou et al.[10]. Their dataset contains 91 images including normal and abnormal CCA which were obtained from SONOS 5500 by Philips Medical System. Their elliptical contours of the CCA were reviewed by clinicians obtaining a JSC of $88 \%$. Lian Luo et al[11], have suggested a method requiring user interface to segment 283 CCA images that contains a weak boundary. The authors obtained the dataset from a Chinese Atherosclerosis study and achieved a JSC of $87 \pm 9 \%$.

Jan Dorazil et al.[12] have suggested a technique for CCA localization using an exhaustive search to find all possible matching centroid of circles in their ROI detection. Their dataset consists of 145 CCA images for healthy people photographed by linear probe L14-5/38 ultrasonic medical system using a frequency of 7 . $5 \mathrm{MHz}$. The authors obtained a mean error of 3.99 and misdetection rates of $0.69 \%$ by comparing their results with the ground truth. Tang et al.[13], suggested a method requiring user intervention to segment the CCA lumen by extracting the center lines of the artery and delineate the boundary of the lumen using level sets. Their proposed technique was trained and tested on open access cls2009 challenge dataset obtaining a JSC of $90.2 \%$.

In this paper, we have proposed an automatic and accurate localization technique to localize the CCA in transverse section US images using Deep learning. We detect the CCA and localize it using Faster RCNN and various localization techniques. The rest of the paper is streamlined as follows: Section 2 presents the methodology. The testing environment is described in section 3. The results and discussion are explained in section 4. Section 5 presents the conclusion and future work.

\section{METHODOLOGY}

We proposed an automated model to localize the CCA in transverse section US images depending on two main steps. First, we detect the rectangular bounding box that contains the CCA in the image using Faster RCNN. Then, we apply various localization techniques to localize the CCA. Fig.1 describes the block diagram of our proposed system.

\subsection{Pre-processing}

We preprocessed both the images used for training and testing of the dataset before entering the proposed system. The preprocessing was applied to the original images of the dataset by downsizing or large sizing the images [14] to be the same size of $227 \times 227 \times 3$. This is to fit the size of the images with the deep learning network used, which is the Faster RCNN containing the AlexNet as its convolutional neural network.

\subsection{CCA bounding box location learning as ground truth}

We used the training Image Labeler Application which was previously built in MATLAB program to define the Region of interest (ROI) [15] by applying a rectangular bounding box around the CCA for each image in our dataset training images and define the category of the object inside the bounding box as the CCA. The bounding box is defined by its left corner (A, B) and its height and width to create a table that serves as a ground truth containing the bounding box coordinates [A, B, width, height]. We used the AlextNet of 23 layers as the CNN of the Faster RCNN. The AlexNet was trained by these bounding boxes which contain the CCA to be fine-tuned and used as a pre-trained network and can identify the location of the CCA.

\subsection{Applying Faster RCNN}




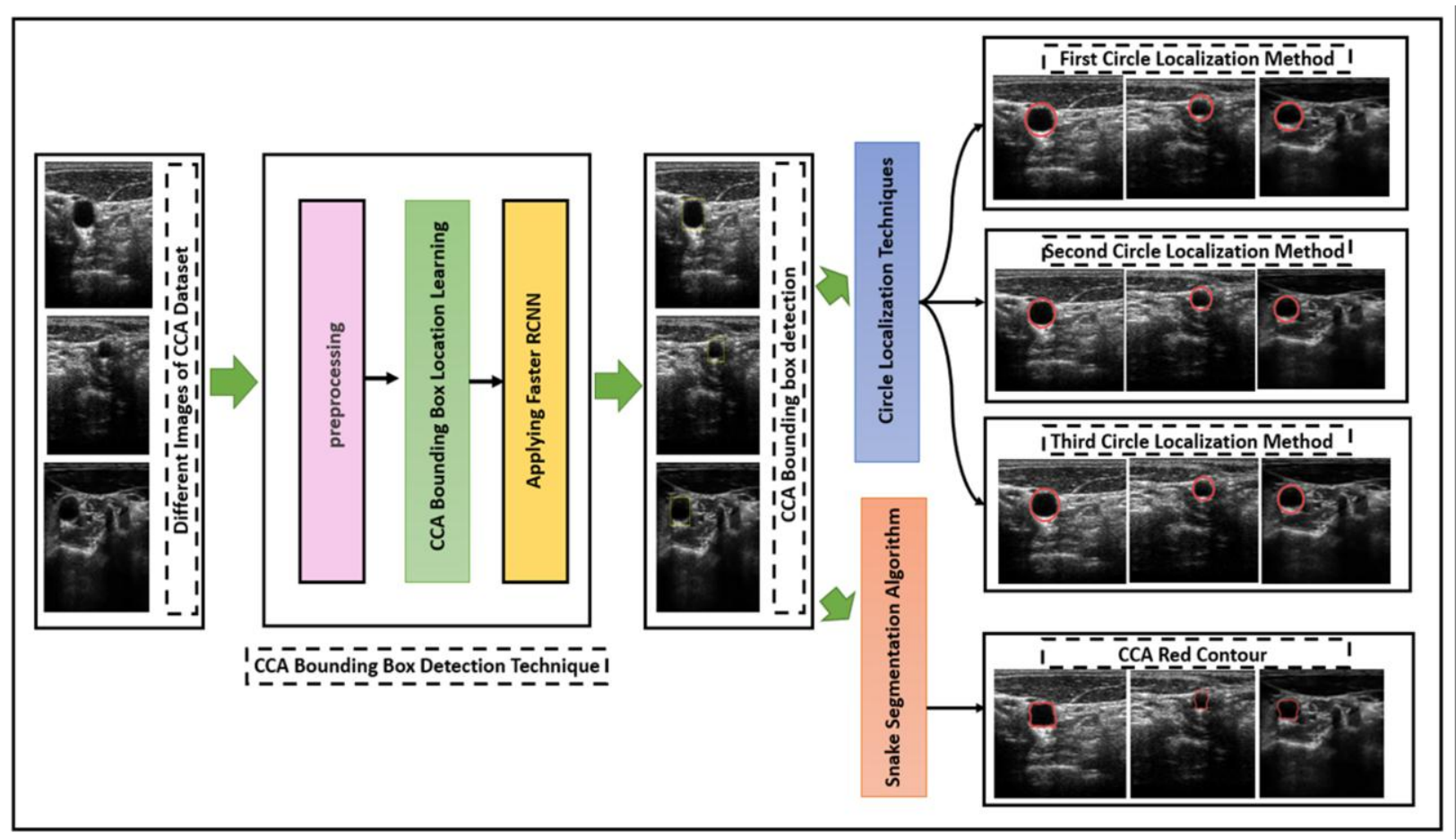

Fig. 1. A flowchart of the proposed CCA localization system contains two main steps. The CCA bounding box detection and CCA circle or contour localization techniques.

Faster RCNN was applied to detect the CCA bounding box efficiently. Faster R-CNN is a deep learning network introduced by Ross Girshick et al.[16] for the object detection process. The faster RCNN consists of a CNN (AlexNet) and a Region proposal Network (RPN) and it is classified as a new iteration of Fast RCNN [17]. The AlexNet extracts the CCA bounding box features to determine the CCA location. The RPN has trained on the CCA bounding box coordinates (anchor) predication and compares its prediction result with the ground truth. The learning and the CCA anchor predictions continue until getting an intersection over union (IOU) in large proportion to the anchors that were marked at the beginning as the ground truth. If the IOU of the anchors and the ground truth are greater than 0.7 , it is considered a positive label and the negative label for IOU is less than 0.3 .

The loss function is described as:

$$
L\left(P_{r}, t_{r}\right)=\frac{1}{N_{s}} \sum_{r} L_{s}\left(P_{r}, P_{r}^{*}\right)+\lambda \frac{1}{N_{g}} \sum_{r} P_{r}^{*} L_{g}\left(t_{r}, t_{r}^{*}\right)
$$

The prediction of the anchor probability and the ground truth is $P_{r}, \mathrm{P}_{\mathrm{r}}{ }^{*}$. The positive label is $\mathrm{t}_{\mathrm{r}}{ }^{*}$. There are four parameterized coordinates saved in $t_{n}$ for the predicted bounding box. The smoothed loss is $\mathrm{L}_{\mathrm{s}}, \mathrm{L}_{\mathrm{g}}$. The mini-batch size $(\mathrm{Ns}=256)$ and the number of anchor locations $(\mathrm{Ng} \sim 2,400)$ and $\lambda=10$. The 4 coordinates for the bounding box regression:

$$
t_{A}=\left(A-A_{a}\right) / C_{a}
$$

$$
\begin{aligned}
& t_{B}=\left(B-B_{a}\right) / D_{a} \\
& t_{C}=\log \left(\frac{C}{C_{a}}\right) \\
& t_{D}=\log \left(D / D_{a}\right) \\
& t_{A}{ }^{*}=\frac{A^{*}-A_{a}}{C_{a}} \\
& t_{B}{ }^{*}=\left(B^{*}-B_{a}\right) / D_{a} \\
& t_{C}{ }^{*}=\log \left(C^{*} / C_{a}\right) \\
& t_{D}{ }^{*}=\log \left(D^{*} / D_{a}\right)
\end{aligned}
$$

(A, B) is the bounding box center. The bounding box width and height are represented in $\mathrm{C}$ and $\mathrm{D}$. The anchor box is $A_{a}$, and $\mathrm{A}^{*}$ is the ground truth box.

\subsection{Localization of the CCA}

After the bounding box detection that contains the CCA using Faster RCNN, we applied various techniques to localize and contour the CCA. The carotid artery was localized in two ways, using several methods to localize the CCA as a circle or using the snake segmentation algorithm to contour the CCA Lumen Intima Boundary (LIB).

\subsubsection{Circle localization methods}

The circle localization techniques of the CCA consist of these essential steps as channel extraction, extracted channel bounding box filling, centroid and diameter 
detection, and CCA circle drawing. We have applied three methods to obtain the artery circle that converges with the clinician's circle and thus gives the highest Jaccard similarity. These methods are similar in channel extraction, extracted channel bounding box filling, centroid detection, and circle drawing but differ in the way of calculating the diameter in each method. First, the channel extraction was applied to extract the yellow detected bounding box obtained by Faster RCNN. Then, we fill the extracted channel of the detected bounding box. After that, a centroid detection was applied to know the bounding box centroid. The rectangular bounding box centroid will be the center point of the circle as (x Centroid, $y$ Centroid) as illustrated in equation (10), (11) where BoundingBox (1), BoundingBox (2), BoundingBox (3), and BoundingBox (4) are the left corner, top corner, width and height of the bounding box. Finally, the

AlgORITHM 1: THE FIRST CIRCLE LOCALIZATION METHOD

Input: CCA image that contains the detecting yellow bounding box

Output: localization of the CCA using a red contour Reading the input image

1. rgblmage $\leftarrow$ imread(folder)

Extracting the individual red, blue and green color channels

2. RedChannel $\leftarrow$ rgblmage $(:,:, 1)$

3. BlueChannel $\leftarrow$ rgblmage $(:,:, 3)$

4. GreenChannel $\leftarrow$ rgblmage $(:,:, 2)$

Getting the yellow detected bounding box and show it

5. yellowDetectedBoundingBox $\leftarrow$ RedChannel $==255 \&$

BlueChannel $==0$ \& GreenChannel $==255$

6. imshow (yellowDetectedBoundingBox)

Extracted channel bounding box filling

7. yellowDetectedBoundingBox $\leftarrow$ imfill( yellowDetectedBoundingBox ,'holes')

8. yelloMask $\leftarrow$ bwareafilt(yelloMask,1)

9. imshow (yellowDetectedBoundingBox )

10. uint8Image $\leftarrow$ uint $8\left(255^{*}\right.$ yellowDetectedBoundingBox )

11. imwrite (uint8Image ,' yellowDetectedBoundingBox ')

Detection of the bounding box centroid and its equivalent diameter

12. props $\leftarrow$ regionprops (yellowDetectedBoundingBox ,'Centroid','EquivDiameter','BoundingBox')

CCA localization with a red contour

13. viscircles (props. Centroid, props. EquivDiameter/2)

CCA was localized by a red circle.

$x$ Centroid $=$ BoundingBox $(1)+$ BoundingBox (3) $/ 2$

$y$ Centroid $=$ BoundingBox $(2)+$ BoundingBox (4)/2

The diameter of the carotid artery circle was calculated using three methods. The first method is to find the equivalent diameter of the circle that has the same area as the rectangular bounding box. The equivalent circular diameter is computed as sqrt (4*Area /pi). The second method is to find the diameter as the sqrt (Area), as the bounding box sides are close in length. The third method is to find the largest side of the rectangle to be the circle diameter. The first circle localization method steps are shown in ALGORITHM 1.

\subsubsection{Snake segmentation}

we applied the snake segmentation algorithm [18] to delineate the CCA LIB. The detected bounding box that was obtained from the Faster R-CNN was used as the initial contour of the snake segmentation algorithm to segment the CCA lumen. The snake contour $\mathrm{k}(\mathrm{c})$ adjusts itself using a dynamic process that reduces the energy function that represents as follows

$$
\begin{aligned}
& \mathrm{E}_{\text {snake }}(k(\mathrm{c}))=\mathrm{E}_{\text {int }}(k(\mathrm{c}))+\mathrm{E}_{\text {image }}(k(\mathrm{c}))+ \\
& \mathrm{E}_{\text {external }}(k(\mathrm{c}))=\int_{\mathrm{c}}\left(\alpha_{\mathrm{c}} \mathrm{E}_{\text {cont }}(k(\mathrm{c}))+\beta_{\mathrm{c}} \mathrm{E}_{\text {curv }}(k(\mathrm{c}))+\right. \\
& \left.\delta_{\mathrm{c}} \mathrm{E}_{\text {image }}(k(\mathrm{c}))+\mathrm{E}_{\text {external }}(k(\mathrm{c}))\right) d c
\end{aligned}
$$

Where $\quad \mathrm{E}_{\text {int }}(\mathrm{k}(\mathrm{c})), \quad \mathrm{E}_{\text {image }}(\mathrm{k}(\mathrm{c})), \quad \mathrm{E}_{\text {cont }}(\mathrm{k}(\mathrm{c}))$, $\mathrm{E}_{\text {external }}(\mathrm{k}(\mathrm{c}))$ and $\mathrm{E}_{\text {curv }}(\mathrm{k}(\mathrm{c}))$ denote the internal, image, continuity, external and curvature of the contour. $\alpha \mathrm{c}$ denotes the strength parameter, $\beta \mathrm{c}$ denotes the tension parameter and $\delta \mathrm{c}$ denotes the stiffness parameter.

\section{TESTING ENVIRONMENT}

\subsection{Machine tool}

Our computations were performed with MATLAB (2018b) using a Laptop with an Intel ${ }^{\circledR}$ Core $^{\mathrm{TM}}$ i3 CPU M380@2.53GHZ Processor with 4 GB RAM.

\subsection{Dataset}

An open-access dataset provided by the SP lab, Brno University of Technology [19] was used to localize the CCA in the transverse mode US images. The dataset was downloaded from the SP Lab site as an m-by-n-by-3 data array. We used 283 images of the CCA in the transverse section obtained from an Ultrasonic OP scanner using a linear probe as 163 images for training and 120 images for testing. The Localization of the CCA in transverse mode has been validated using the SP lab ground truth which contains the center and radius of the Media-Adventitia Boundary (MAB) circle of the CCA.

\section{Results and Discussion}

\subsection{Evaluation metrics}

We have been quantified the performance of the CCA localization by calculating the JSC for our proposed system resulting circle or contour and the clinicians' circles to assess the efficiency of our proposed system as described in equation (13), respectively [20].

$$
J S C(Q, R)=\frac{|Q \cap R|}{|Q \cup R|}
$$


Where $\mathrm{Q}$ is the experts' manual circle and $\mathrm{R}$ is our proposed system resulting circle or contour, respectively.

\subsection{Results}

We introduced an automatic and accurate CCA localization system because this is a very important step to predict and detect CCA hardening. Carotid artery occlusion is a slow and progressive disease that may begin in early childhood. So early diagnosis contributes to stopping atherosclerosis from getting worse and thus preventing exposure to a heart attack, stroke, or medical emergency.

So, we proposed a CCA localization system that contains two main phases as the CCA bounding box detection technique and various localization techniques of the CCA. The CCA bounding box detection was applied using Faster RCNN. We have achieved correct CCA bounding boxes in the most utmost cases. Nevertheless, in a diminutive number of cases, we didn't get the required CCA bounding box as output. The CCA detection accuracy is computed as the percentage of images in which the CCA bounding box has been successfully detected in the dataset. We have achieved a CCA detection accuracy of $97.5 \%$ which indicates the CCA bounding boxes that have correctly detected.

The circle localization methods and the snake segmentation output have been evaluated by calculating the JSC between our proposed methods as the circles or contours and the clinicians' manual circles. The first circle localization method achieved a JSC of $90.86 \%$ which showed a great match between our system and the doctor's manual delineations. The second circle localization method achieved a JSC of $82.54 \%$. The third circle localization method achieved a JSC of $89.58 \%$. The snake segmentation contours achieved a JSC of $67.72 \%$. The JSC of our various methods to localize the CCA was shown in Table 1.

The first circle localization method gave much better results than other methods and other researchers. This method got numbers very close to the standard clinicians' values. Table 2 reviews the performance of our proposed method in comparison with other systems showing that our system is superior to others introduced in the literature.

Table 1

RESULTS OF THE VARIOUS METHODS USED FOR THE CCA LOCALIZATION IN THE TRANSVERSE MODE

\begin{tabular}{lc}
\hline \multicolumn{1}{c}{ Method } & (Jaccard similarity) \\
\hline First circle localization method & $\mathbf{9 0 . 8 6}$ \\
Second circle localization method & 82.54 \\
Third circle localization method & 89.58 \\
Snake segmentation algorithm & 67.72 \\
\hline \hline
\end{tabular}

Table 2

COMPARATIVE ANALYSIS OF VARIOUS ALGORITHMS FOR CCA LOCALIZATION IN THE TRANSVERSE MODE

\begin{tabular}{cccc}
\multicolumn{4}{c}{ LOCALIZATION IN THE TRANSVERSE MODE } \\
\hline \hline Method & $\#$ & (Jaccard - & Type \\
& image & similarity) & Type \\
\hline
\end{tabular}

\begin{tabular}{lccc}
\hline Yang et al. [6] & 180 & 70.5 & Automated \\
Yang et al. [7] & 110 & 90.3 & Semi-automated \\
Hamou et al. [10] & 91 & 88 & Automated \\
Lian Luo et al. & 283 & $87+9$ & Semi-automated \\
[11] & & & \\
$\begin{array}{l}\text { Tang et al. [13] } \\
\begin{array}{l}\text { Proposed } \\
\text { Method }\end{array}\end{array}$ & 56 & 90.2 & Semi-automated \\
\hline \hline
\end{tabular}

The CCA bounding box location was learned using
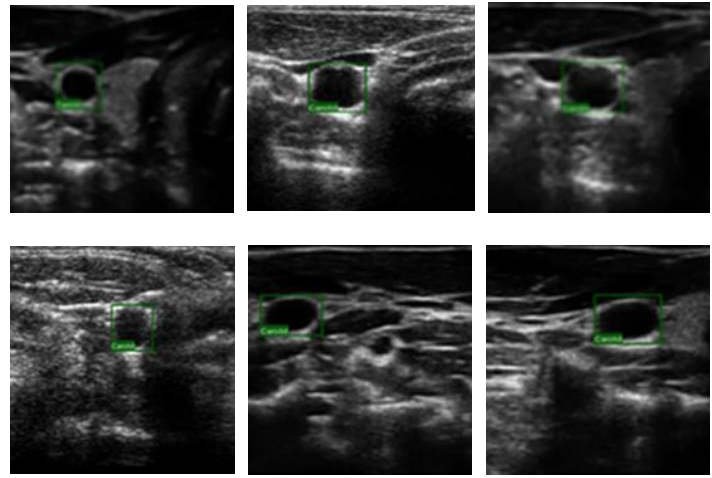

Fig. 2. Applying a rectangular bounding box around the RoI and classify the ROI as "Carotid" for each image of the training images of the dataset to serve as aground truth.
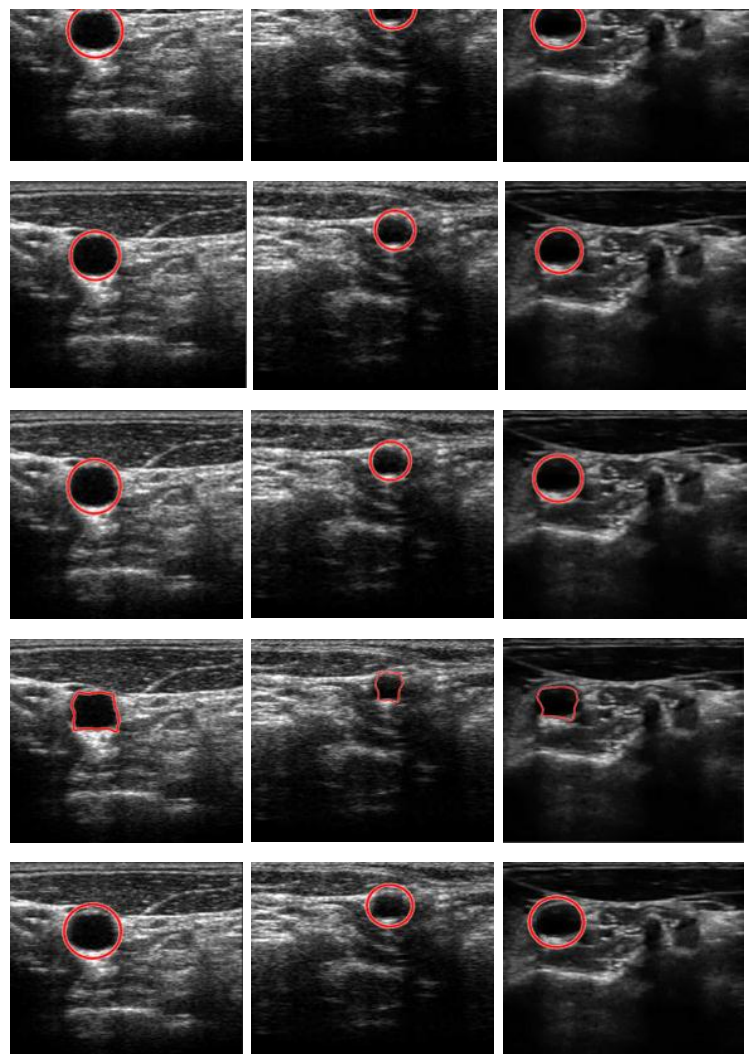

Fig. 3. The localization of CCA in transverse mode. The first row contains the original image. The second row contains the detected bounding box around the CCA using Faster R-CNN. The third row provides the red circle of the CCA using the first circle localization method. The fourth row provides the red circle of the CCA using the second circle localization method. The fifth row provides the red circle of the CCA using the third circle localization method. The sixth row provides the red contour of the CCA using the snake segmentation algorithm. The seventh row provides the clinicians' circle. 


\section{Vol. 40, No. 2. July 2021}

the Image Labeler Application. This is done by defining the ROI by implementing a rectangular bounding box surrounding the CCA and label the category of the object inside the bounding box as the CCA as shown in Fig. 2. The results of our CCA localization systems in transverse mode are shown in Fig. 3.

The first row of Fig. 3 includes different samples of the original image of the SP lab dataset [19]. The second row includes the CCA detected bounding boxes that have correctly recognized after applying the Faster R-CNN. The third row displays the CCA circle using the first circle localization method. The fourth row displays the CCA circle using the second circle localization method. The fifth row displays the CCA circle using the third circle localization method. The sixth row provides the CCA red contour using the snake segmentation algorithm. The seventh row provides the clinicians' circle for each image.

\subsection{Discussion}

In this paper, we have proposed a system for automatic and accurate localization of the carotid artery because it is classified from silent diseases that can cause stroke or paralysis suddenly without prior warning. Carotid artery disease is one of the most important causes of stroke, as it is responsible for supplying the brain with oxygenated blood. Therefore, people who are at high risk of developing atherosclerosis should perform a periodic examination of the carotid artery for early treatment to avoid serious health problems.

The CCA localization system was applied using the CCA bounding box detection and the circle localization techniques to localize the CCA. This is used as a strong indication and evidence of any simple change that occurs to the artery or the beginning of the accumulation of calcium or fatty deposits and thus taking the necessary medical action without entering into medical complications.

The bounding box detection technique was utilized using Faster RCNN that contains AlexNet and RPN inside it to determine the location of the CCA in the image. The resizing step is an essential preprocessing step and is used to assist in the automatic detection of the CCA using Faster RCNN. We used the AlexNet as the CNN of the Faster RCNN because it is distinguished from other networks in that it is trained faster and limited the overfitting. The AlexNet has 23 layers and (227 $\times 227 \times 3)$ images were applied in its input layer. we pre-trained the AlexNet on our dataset images to determine the CCA location by extracting the features of the CCA bounding boxes. The RPN has trained using the CCA bounding box coordinates to predict it correctly. The RPN training process continues to reach the closely matched bounding box with the ground truth.

The CCA detection model was trained for just 10 epochs and the model attested very good performance. The estimated detection time is roughly 0.2 seconds per each separate image. The evaluations for Faster RCNN are implemented based on the non-maximum suppression (NMS) parameter threshold which is used to lessen the redundancy on proposed regions.

Our system was distinguished from other systems for obtaining enormously better results than other states of the arts and getting values that are very adjacent to the standard clinicians' diagnosis. The CCA bounding box detection accuracy for identifying the CCA is $97.5 \%$, this percentage means the number of the dataset images in which the CCA bounding box was correctly identified. Also, our system obtained a JSC of $90.86 \%$ by applying a comparison between our circles and the clinical circles which are given as the center and radius of the MAB of the CCA circles. This percentage has surpassed other researches that existed in the literature as shown in Table $r$. We used the clinician's manual circles as the ground truth to validate our results.

Our proposed system is fully automated and has the highest similarity with the clinician's standard results. Unlike the other state of the arts that used a semiautomated method in their proposed system which requires user interaction as Yang et al [7] suggested a semi-automated segmentation procedure to contour the CCA using the GVF-Snake model and morphological operations. A method involving a user interface to segment the CCA images that contain a weak boundary has been proposed by Lian Luo et al [11].

A semiautomatic segmentation technique focused on deep learning to segment the carotid ultrasound images was suggested by Ran Zhou et al. [21] to identify the patches created by sliding a window along the standard line of the initial contour where the CNN model is finetuned in each test task. The carotid is segmented by adding a region of interest of carotid images to a U-Net model enabling the network to be trained for pixel-wise classification end-to-end. Tang et al. [13] proposed a procedure involving user interference to segment the CCA lumen by extracting the artery's center lines and using level sets to delineate the boundaries of the lumen.

Some studies have targeted the segmentation of wall interfaces for transverse carotid ultrasound images [22], [23] but they didn't thoroughly address the manual tuning issue to achieve reasonable segmentation results. In this paper, we used the faster RCNN to get the initial rectangular contour automatically by fine-tuning the pre-trained AlexNet to identify the location of the CCA and delineate the artery circle.

we have applied several methods to obtain the largest percentage of intersection between our circle and the clinicians' circle. The point here is to determine the media adventitia boundary of the common carotid artery as the clinicians define the CCA circles. Therefore, the best method for localizing the CCA that has the highest JSC is when using the equivalent diameter of the first localization method.

The carotid contours resulting from the snake segmentation algorithm obtained the lowest Jaccard similarity by comparing them with clinicians' circles because the contour determined the lumen intima boundary, but it is expected that this method will achieve superior results in the case of determining the lumen of the artery in the transverse section. Also, the 


\section{Vol. 40, No. 2. July 2021}

snake segmentation method will provide accurate delineation of the artery in the cases of plaque because it will be able to accurately track the artery lumen as it is providing irregular shapes.

We aim to achieve automatic localization and contouring in cases of plaque and the carotid longitudinal section in future research developments to make a complete diagnostic system for carotid artery disease. The automatic localization will be achieved by applying the first stage of our proposed system. This stage will contribute to the automatic determination of the location of the bounding box, whether in the longitudinal section or the case of plaque. Then we will apply the snake segmentation algorithm to trace over the occluded areas precisely and accurately delineate the CCA in the longitudinal section.

In this paper, we obtained the initial contour automatically and applied a snake segmentation method that provides irregular shapes to accurately follow the longitudinal section of the artery or the plaque.

Unlike the other state of the arts that select the initial bounding box manually as Nirvedh $\mathrm{H}$. Meshram et al.[24] proposed a semi-automatic segmentation approach that involves the input of a sonographer to provide limited inputs, such as bounding boxes or seed points, to achieve reasonable segmentation output on these carotid B-mode images in patients with a substantial plaque and related shadowing artifacts. Destrempes et al. [25] provided a system by which the user got a manual segmentation of the carotid plaque according to which motion prediction and the Bayesian model were used to approximate plaque boundary. Also, a method was developed by McCormick et al. [26] for the manual segmentation of the plaque at the diastole image using the segmented occluded area monitored over the rest of the cardiac cycle with a displacement estimation and using a multi-level approach and Bayesian regularization. Zhou et al. [27] utilized U-Net for the plaque segmentation in the carotid transverse mode by providing the network with data that were segmented with an initial contour of media-adventitia and lumen-media boundaries, following which a neural network to segment the plaque.

\section{Conclusion and future work}

An automated and accurate system was proposed to localize the CCA in transverse section ultrasound (US) images using deep learning. Preprocessing was applied to the input dataset images to be ready for the deep learning network. The Deep learning network is represented in using the faster RCNN to detect the CCA bounding box. After the CCA bounding box detection, various localization techniques were applied to localize the CCA. In the future, we aim to apply a complete diagnostic system for the CCA disease. We aim to apply our proposed system to the longitudinal section of the carotid artery but there was no available dataset that contains sufficient images of the carotid artery in the longitudinal section. Also, there is a dearth of databases containing artery occlusion or plaque CCA. so, we aim to generate a standard database containing the CCA images in longitudinal mode and sufficient images for longitudinal and transverse of an atherosclerotic artery to apply the complete diagnostic system.

\section{References}

[1] L. C. Sousa et al., "Toward hemodynamic diagnosis of carotid artery stenosis based on ultrasound image data and computational modeling," Medical \& biological engineering \& computing, vol. 52, no. 11, pp. 971-983, 2014.

[2] S. S. Virani et al., "Heart disease and stroke statistics-2020 update: a report from the American Heart Association," Circulation, pp. E139-E596, 2020.

[3] J. W. Tenney et al., "Retrospective evaluation of resuscitation medication utilization in hospitalized adult patients with cardiac arrest," Journal of Cardiology, 2020.

[4] M. Lou et al., "Chinese Stroke Association guidelines for clinical management of cerebrovascular disorders," Stroke and Vascular Neurology, pp. svn-2020-000355, 2020.

[5] S. Latha, D. Samiappan, and R. Kumar, "Carotid artery ultrasound image analysis: A review of the literature," Proceedings of the Institution of Mechanical Engineers, Part H: Journal of Engineering in Medicine, vol. 234, no. 5, pp. 417-443, 2020.

[6] X. Yang, M. Ding, L. Lou, M. Yuchi, Q. Wu, and Y. Sun, "Common carotid artery lumen segmentation in B-mode ultrasound transverse view images," International Journal of Image, Graphics and Signal Processing, vol. 3, no. 5, p. 15, 2011.

[7] X. Yang, W. He, J. Jin, X. Zhang, M. Yuchi, and M. Ding, "A hybrid method to segment common carotid arteries from 3D ultrasound images," in Proceedings of 2012 IEEE-EMBS International Conference on Biomedical and Health Informatics, 2012, pp. 241-244: IEEE.

[8] R. Benes, J. Karasek, R. Burget, and K. Ríha, "Automatically designed machine vision system for the localization of CCA transverse section in ultrasound images," Computer methods and programs in biomedicine, vol. 109 1, pp. 92-103, 2013.

[9] S. Singh and A. K. Sahani, "A Machine Learning Approach to Carotid Wall Localization in A-mode Ultrasound," in 2020 IEEE International Symposium on Medical Measurements and Applications (MeMeA), 2020, pp. 1-5: IEEE.

[10] A. K. Hamou, S. Osman, and M. R. El-Sakka, "Carotid ultrasound segmentation using DP active contours," in International Conference Image Analysis and Recognition, 2007, pp. 961-971: Springer. 
Vol. 40, No. 2. July 2021

[11] L. Luo et al., "Carotid artery segmentation using level set method with double adaptive threshold (DATLS) on TOF-MRA images," Magnetic resonance imaging, vol. 63, pp. 123-130, 2019.

[12] J. Dorazil, K. Ríha, and M. K. Dutta, "Common Carotid Artery Wall Localization in B-mode Ultrasound Images for Initialization of Artery Wall Tracking Methods," 2019 42nd International Conference on Telecommunications and Signal Processing (TSP), pp. 605-608, 2019.

[13] H. Tang, T. van Walsum, R. Hameeteman, R. Shahzad, L. J. van Vliet, and W. J. Niessen, "Lumen segmentation and stenosis quantification of atherosclerotic carotid arteries in CTA utilizing a centerline intensity prior," Medical physics, vol. 40, no. 5, p. 051721, 2013.

[14] D. A. Sultan, "Image resizing with minimum distortion," Periodicals of Engineering and Natural Sciences, vol. 8, no. 2, pp. 763-772, 2020.

[15] A. Islam, P. Saha, M. Rana, M. M. Adnan, and B. B. Pathik, "Smart Gardening Assistance System with the Capability of Detecting Leaf Disease in MATLAB," in 2019 IEEE Pune Section International Conference (PuneCon), 2019, pp. 1-6.

[16] S. Ren, K. He, R. B. Girshick, and J. Sun, "Faster R-CNN: Towards Real-Time Object Detection with Region Proposal Networks," IEEE Transactions on Pattern Analysis and Machine Intelligence, vol. 39, pp. 1137-1149, 2015.

[17] R. B. Girshick, "Fast R-CNN," 2015 IEEE International Conference on Computer Vision (ICCV), pp. 1440-1448, 2015.

[18] D. J. Williams and M. Shah, "A Fast algorithm for active contours and curvature estimation," CVGIP: Image Understanding, vol. 55, no. 1, pp. 14-26, 1992/01/01/ 1992.

[19] K. M. ŘÍHA, J.; BURGET, R.; BENEŠ, R.; ZÁVODNÁ, E. Artery databases [Online]. Available:

http://splab.cz/en/research/zpracovani-

medicinskych-signalu/databaze/artery

[20] S. Kabir, C. Wagner, T. C. Havens, and D. T. Anderson, "A Similarity Measure Based on Bidirectional Subsethood for Intervals," IEEE Transactions on Fuzzy Systems, pp. 1-1, 2020.

[21] R. Zhou, A. Fenster, Y. Xia, J. D. Spence, and M. Ding, "Deep learning- based carotid media- adventitia and lumen- intima boundary segmentation from threedimensional ultrasound images," Medical physics, vol. 46, no. 7, pp. 3180-3193, 2019.

[22] E. Ukwatta et al., "Three- dimensional ultrasound of carotid atherosclerosis: semiautomated segmentation using a level set- based method," Medical physics, vol. 38, no. 5, pp. 2479-2493, 2011.
[23] J. C. Seabra, L. M. Pedro, J. F. e Fernandes, and J. M. Sanches, "A 3-D ultrasound-based framework to characterize the echo morphology of carotid plaques," IEEE Transactions on Biomedical Engineering, vol. 56, no. 5, pp. 1442-1453, 2009.

[24] N. H. Meshram, C. C. Mitchell, S. Wilbrand, R. J. Dempsey, and T. Varghese, "Deep Learning for Carotid Plaque Segmentation using a Dilated U-Net Architecture," Ultrasonic Imaging, vol. 42, no. 4-5, pp. 221230, 2020.

[25] F. Destrempes, J. Meunier, M.-F. Giroux, G. Soulez, and G. Cloutier, "Segmentation of plaques in sequences of ultrasonic B-mode images of carotid arteries based on motion estimation and a Bayesian model," IEEE Transactions on Biomedical Engineering, vol. 58, no. 8, pp. 2202-2211, 2011.

[26] M. McCormick, T. Varghese, X. Wang, C. Mitchell, M. Kliewer, and R. Dempsey, "Methods for robust in vivo strain estimation in the carotid artery," Physics in Medicine \& Biology, vol. 57, no. 22, p. 7329, 2012.

[27] R. Zhou, W. Ma, A. Fenster, and M. Ding, "U-Net based automatic carotid plaque segmentation from 3D ultrasound images," in Medical Imaging 2019: Computer-Aided Diagnosis, 2019, vol. 10950, p. 109504F: International Society for Optics and Photonics. 
Vol. 40, No. 2. July 2021

التدايد التلقائي للشريان السباتي الرئيسي في صور الموجات فوق الصوتية باستخام تقنيات التعلم العميق

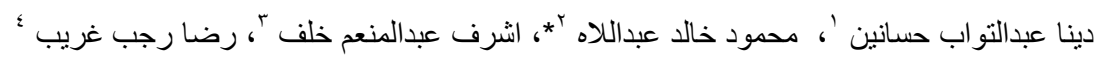

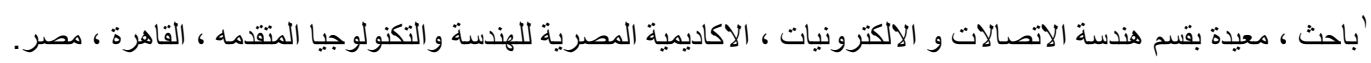

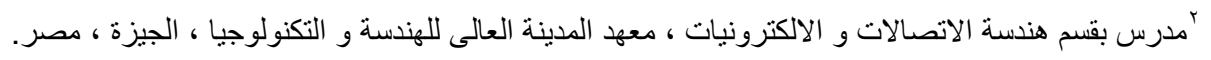

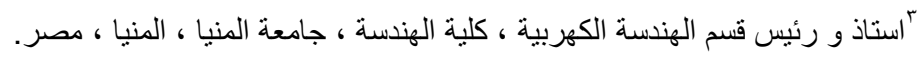

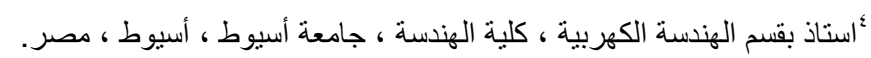

الملخص :

يعد مرض الثريان السباتي من الامر اض الصامته الذي لا يسبب اي اعراض في مراحله المبكره ، و غالبا لا يدرك الاشخاص أنهم يعانون من مشكلة حتى يصابوا بالسكتة الدماغية. تُعرف الثرايين السباتية بأنها الأوعية الدموية الرئيسية في الرقبة التي تزود المخ والرقبة والوجه بالدم. يحدث انسداد الثريان السباتي بسبب ارتفاع نسبة الكوليسترول في الدم وتر اكم المواد الدهنية على جدران الأوعية الدموية ، مما يتسبب في ضيق الثريان الذي يؤدي تري الي اعاقة تدفق الدم إلى المخ وحدوث تصلب للثريان السباتي و السكته الدماغية. يؤدي تلف المخ الناتج عن السكتة الدماغية إلى فقدان الكلام أو الرؤية أو الثلل، ويمكن أن تؤدي السكتات الدماغية الثديدة إلى الوفاة؛ فالسكتة الدماغية هي ثاني أهم سبب للوفاة في العالم بعد أمراض القلب. الهدف الرئيسي من هذا البحث هو التحديد التلقيأي والدقيق للثريان السباتي الرئيسي في صور الموجات فوق الصوتية ذات المقطع العرضي باستخدام تقنيات التعلم العميق وذلك لاجراء التدخل الطبي اللازم دون الدخول في الدضاعفات الصحية والعلاج قبل حدوث السكتة الدماغية. أولاً ، قمنا بتجهيز الصور وذلك بتطبيق المعالجة المسبقة على الصور الأصلية لهجموعة البيانات عن طريق تصغير الحجم أو تكبيره ليتناسب حجم الصور مع شبكة التعلم العميق المستخدمة. ثانيا ، استخدمنا شبكه التعلم العميق (Faster RCNN) لاكتشاف المربع المحيط بالثريان السباتي. ثالثا ، طبقنا تقنية لتحديد دائرة الثريان السباتي اعتمادا علي


بنسبه r^. •9\% وذلك بالمقارنة بين نتائجنا ونتائج الأطباء. تم استخدام قاعده البيانات الففتوحه التي يقدمها مختبر معالجه الاشارات وتم تطبيق نظامنا المقترح علي برنامج الماتلاب ـ أظهر نظامنا المقترح تفوق كبير عند مقارنته بالأنظمة الأخرى . 